\title{
Revisiting Metallicity of Long Gamma-Ray Burst Host Galaxies: The Role of Chemical Inhomogeneities in Galaxies
}

\author{
Yuu Niino \\ Division of Optical and NIR Astronomy, National Astronomical Observatory of Japan \\ 2-21-1 Osawa, Mitaka, Tokyo, Japan email: yuu.niino@nao.ac.jp
}

\begin{abstract}
Some theoretical studies on the origin of long gamma-ray bursts (GRBs) using stellar evolution models suggest that a low metallicity environment may be a necessary condition for a GRB to occur. However, recent discoveries of high-metallicity host galaxies of some GRBs cast doubt on the requirement of low-metallicity in GRB occurrence. In this study, we predict the metallicity distribution of GRB host galaxies, assuming empirical formulations of galaxy properties. We take internal dispersion of metallicity within each galaxy into account. Assuming GRBs trace low-metallicity star formation $12+\log (\mathrm{O} / \mathrm{H})<8.2$, we find that $\gtrsim 10 \%$ of GRB host galaxies may have $Z>Z_{\odot}$, depending on the internal dispersion of metallicity within galaxies.
\end{abstract}

Keywords. gamma rays: bursts, galaxies: abundances

\section{Models of Galaxies}

We assume following empirical formulations of galaxy properties at $z \sim 0.1$, to compute expected $M_{\star}$ and metallicity distribution of GRB host galaxies.

- Stellar mass function (Bell et al. 2003; Drory \& Alverez 2008)

- Star formation rate (SFR) as a function of $M_{\star}$ (Brinchmann et al. 2004; Stanek et al. 2006; Drory \& Alverez 2008)

- $M_{\star}-Z$ relation (Savaglio et al. 2005, recalibration of SDSS sample with R23 method) Dispersion of SFR and $M_{\star}-Z$ relation is $\sigma=0.3 \& 0.1$ dex, respectively.

Metallicity of GRB host galaxies is an important clue to study the metallicity of GRB progenitors. However metallicity of a galaxy is not necessarily equal to metallicity of young stars formed in the galaxy. The Milky Way (MW) has mean metallicity $12+\log (\mathrm{O} / \mathrm{H}) \sim 8.9$, but some young B-type stars and HII regions in the Milky Way have $12+\log (\mathrm{O} / \mathrm{H}) \sim 8.2$ (e.g. Afflerbach et al. 1997; Rolleston et al. 2000).

Motivated by the observations of galactic B-type main sequence stars and HII regions, we consider internal metallicity distribution of young stars within each galaxy with log-normal tail $\left(\sigma_{Z, \text { int }}=0.1,0.3 \& 0.5\right)$. In each galaxy, we assume $R_{\mathrm{GRB}} \propto$

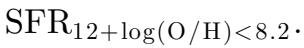

\section{Results}

The computed metallicity distribution is shown in figure 1. No metallicity preference model contradicts observations as suggested by Stanek et al. (2006). In $\sigma_{Z \text {,int }}=0.3,0.5$ models, high-metallicity galaxies with $12+\log (\mathrm{O} / \mathrm{H})>8.8$ contribute $5-25 \%$ of cosmic low-metal star formation $[12+\log (\mathrm{O} / \mathrm{H})<8.2]$, or GRB rate. Double peak distribution of host metallicity appears when $\sigma_{Z \text {,int }}$ positively correlates with $M_{\star}$. 


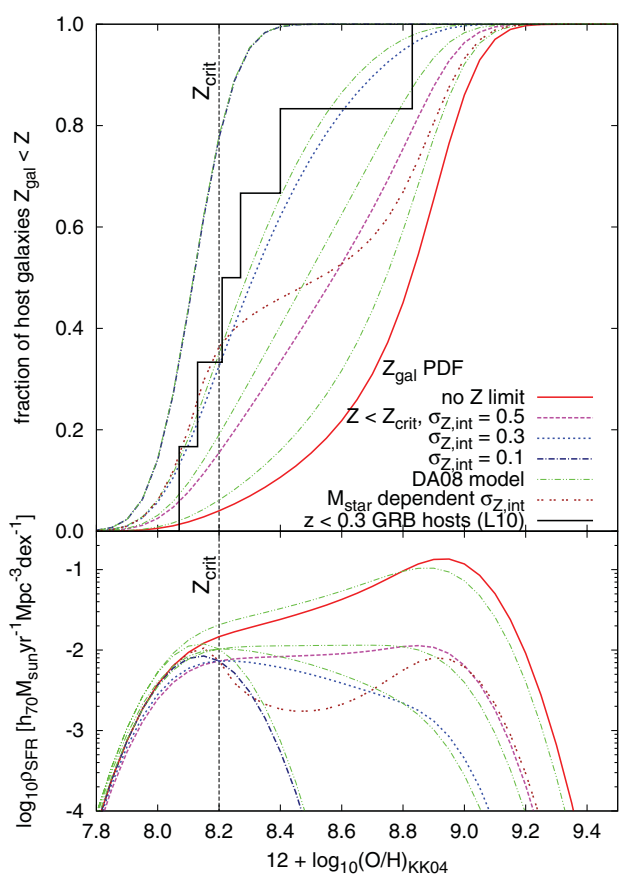

Figure 1. Metallicity distribution of GRB host galaxies predicted in our model compared to current sample collected by Levesque et al. (2010).

\section{Discussion}

We have computed the expected metallicity distribution of GRB host galaxies assuming empirical formulations of galaxy properties at $z \sim 0.1$ and the model of GRB rate in which GRBs occur only from low-metallicity stars. Our results show that high-metallicity galaxies $[12+\log (\mathrm{O} / \mathrm{H})>8.8]$ may have significant contribution to cosmic GRB rate. This means that metallicities of GRB host galaxies may be systematically different from those of GRB progenitors, and the low-metallicity scenario can be reconciled with the observations of high-metallicity host galaxies of GRBs. More detailed discussion is in Niino (2011).

\section{References}

Afflerbach, A., Churchwell, E., \& Werner, M. W. 1997, ApJ, 478, 190

Bell, E. F., McIntosh, D. H., Katz, N., \& Weinberg, M. D. 2003, ApJS, 149, 289

Brinchmann, J., Charlot, S., White, S. D. M., Tremonti, C., Kauffmann, G., Heckman, T., \& Brinkmann, J. 2004, MNRAS, 351, 1151

Drory, N. \& Alvarez, M. 2008, ApJ, 680, 41

Levesque, E. M., Kewley, L. J., Berger, E., \& Jabran Zahid, H. 2010, AJ, 140, 1557

Niino, Y. 2011, MNRAS, 417, 567

Rolleston, W. R. J., Smartt, S. J., Dufton, P. L., \& Ryans, R. S. I. 2000, A\&A, 363, 537

Savaglio, S. et al. 2005, ApJ, 635, 260

Stanek, K. Z. et al. 2006, AcA., 56, 333 